Health-IT

\section{Gegenüber echten Ärzten sehen Diagnoseprogramme alt aus}

\author{
Computerbasierte Algorithmen zur Symptomanalyse gelten \\ als Mittel, ärztliche Diagnosefehler zu vermeiden. Doch bis es \\ so weit ist, dauert es wohl noch ein Weilchen.
}

\begin{abstract}
In allen Bereichen des menschlichen Lebens, in denen es Fehler auszumerzen gilt, kommen im ITZeitalter computergestützte Algorithmen als vermeintliche Problemlöser ins Spiel. Die Medizin bildet da keine Ausnahme: Auch hier passieren Fehler, und jeder Versuch einer Elimination ist aller Ehren wert. So existiert bereits eine Fülle von Programmen, die nach Eingabe von Symptomen eine (Differenzial-)Diagnose ausspucken.

Forscher der Harvard Medical School haben nun 23 solcher „Symptom-Checker" gegen 234 Ärzten (90\% Internisten)

der Fälle vertreten. Besonders armselig schnitten die Computeralgorithmen bei den hochakuten Erkrankungen ab: Während die richtige Diagnose bei den Ärzten in $79 \%$ der Fälle die Hauptdiagnose und in $89 \%$ unter den ersten drei Differenzialdiagnosen war, erreichten die program-

mierten Checker hier nur Raten von $24 \%$ und $40 \%$.

Bei den häufigen Erkrankungen
\end{abstract} antreten lassen. $\mathrm{Zu}$ lösen waren 45 Fälle in Form klinischer Vignetten. Es gab Krankheitsbilder verschiedener Häufigund Dringlichkeit, vom Leberversagen über die Mittelohrentzündung bis zur Candidainfektion.

\section{Fleisch-Ärzte gewinnen mit $72: 34$}

Für jeden Fall sollten die vermuteten Differenzialdiagnosen nach ihrer Wahrscheinlichkeit aufgelistet werden. Die Trefferquote der Ärzte in puncto Hauptdiagnose betrug $72 \%$, die der SymptomChecker nur 34\%. Betrachtete man die an den ersten drei Stellen genannten Differenzialdiagnosen, war die korrekte Diagnose bei den Medizinern in $84 \%$ und bei den Symptom-Checkern in 51\% hatten Ärzte in 70\% der Fälle die richtige Diagnose an Position eins und in 83\% unter den ersten drei. Die Checker erreichten hier 38\% bzw. 56\%. Für seltene Krankheitsbilder betrugen die Quoten für die Ärzte 76\% bzw. 86\% und für die Algorithmen 28\% bzw. 45\%.

Damit liegen die Ärzte beim diagnostischen Leistungsvermögen gemäß den Studienergebnissen weit vor den softwarebasierten Diagnosewerkzeugen. Die Forscher spekulieren aber, dass sich die Rate der korrekten Diagnosen durch eine Kooperation von Arzt und Rechner noch steigern ließe - vielleicht auch schon in der näheren Zukunft. - r rb

- Semigran HL et al. JAMA Intern Med 2016, online 10. Oktober; doi: 10.1001/jamainternmed.2016.6001
Hormonpräparate

\section{Kontrazeption kann depressiv machen}

_ Der Zusammenhang zwischen hormoneller Kontrazeption und Depression wurde an der Universität Kopenhagen anhand von zwei landesweiten Registern für Arzneimittelverordnungen bzw. für Entlassungsdiagnosen psychiatrischer Kliniken untersucht. So konnten mehr als eine Million Frauen und Mädchen zwischen 15 und $34 \mathrm{im}$ Mittel über sechs Jahre verfolgt werden.

55,5\% der Frauen hatten in dieser Zeit hormonelle Verhütungsmittel genutzt. Pro 100 Personenjahre wurde 2,2 von ihnen erstmalig ein Antidepressivum verordnet und bei 0,3 erstmals eine Depression diagnostiziert. Bei Frauen, die in den letzten sechs Monaten nicht mit Hormonpräparaten verhütet hatten, betraf dies nur 1,7 bzw. 0,28.

\section{Risikosteigerung bis zu $\mathbf{1 1 0} \%$}

Das Risiko, ein Antidepressivum verschrieben zu bekommen, war bei der Einnahme kombinierter oraler Kontrazeptiva (inklusive modernerer Präparate mit niedrigen Ethinylestradioldosen und neueren Gestagenen) um 20\% erhöht, bei reinen Gestagenpillen um 30\%, bei transdermalen Pflastern um $100 \%$, bei Vaginalringen um $60 \%$, bei Intrauterinpessaren um $40 \%$ und bei Hormonimplantaten um 110\%. Die Exzessrisiken für eine Depressionsdiagnose lagen etwas niedriger.

Mit dem Alter sank das Risiko, unter einer Hormonanwendung depressiv zu werden. Am stärksten gefährdet waren Mädchen zwischen 15 und 19 Jahren. Auch die Dauer des Hormongebrauchs war ein Einflussfaktor: Das höchste Depressionsrisiko bestand nach sechsmonatiger Anwendung, danach ging es langsam wieder zurück. 